\title{
Male Genital System Fluid or Secretion
}

National Cancer Institute

\section{Source}

National Cancer Institute. Male Genital System Fluid or Secretion. NCI Thesaurus. Code C13713.

Sperm and the fluid it is suspended in. 